\title{
A Novel Hybrid Model for Automated Analysis Of Cardiotocograms Using Machine Learning Algorithms
}

\author{
Emre Avuçlu*1
}

\begin{abstract}
Submitted: 11/10/2021 Accepted : 17/12/2021
Abstract: In this study, a new hybrid model was presented for the prediction of fetal state from fetal heart rate (FHR) and the uterine contraction (UC) signals obtained from cardiotocogram (CTG) recordings. CTG monitoring of FHR and uterine contractions during pregnancy and delivery provides information on the physiological status of the fetus to identify hypoxia. The precise information obtained from these records provides some ideas for interpreting the pathological condition of the fetus. Thus, with early intervention, it allows to prevent any negative situation that will occur in the fetus in the future. In this study, due to the importance of this subject, a new hybrid model was developed which can perform high rate accurate diagnosis using Machine Learning (ML) algorithms. In the hybrid model, 4 different ML algorithms (k Nearest Neighbors (k-NN), Decision Tree (DT), Naive Bayes (NB) and Support Vector Machine (SVM)) were used. While the diagnosis without the hybrid model was low, the improved hybrid model increased the accuracy by $34 \%$. As a result of this hybrid model, $100 \%$ success was achieved for classification, test success, Accuracy, Sensitivity and Specificity with NB and DT ML algorithms.
\end{abstract}

Keywords: Biomedical diagnostics, Machine Learning algorithms, Fetal heart rate measurement.

This is an open access article under the CC BY-SA 4.0 license. (https://creativecommons.org/licenses/by-sa/4.0/)

\section{Introduction}

The FHR detection module is used to detect the heart sound of the unborn baby. FHR detection module: The transmitter and receiver, called Tx and Rx, consist of ultrasonic sensors and a modulator and demodulator circuit controlling these ultrasonic sensors. FHR is a method used to control the fetus's state of health in the womb. FHR is also used when there are birth pains and during labor. The FHR can provide information on the health status of the fetus to doctors and provide precautionary measures. Particularly in dangerous pregnancies, FHR is more important [1]. Ultrasonic sensors are mainly used to convert the applied electrical signal to an audio signal or to convert the sound signal impinging on the sensor to an electrical signal. The signal is passed through a series of electronic filters so that the heart sound signal detected from the Rx ultrasonic sensor can be removed from the accompanying electrical noise. The cleaned signal is the electrical signal of the heart sound reflected by the movements of the heart and detected by ultrasonic sensors. This electrical signal corresponds to an electrical signal value of very small amplitude to be detected by the microprocessor. The electronic amplifier passes through the circuit stage to raise the electrical signal to an electrical signal level that can be detected by the microprocessor. Thus, the electrical signal is increased to a level that can be detected by the microprocessor. At the same time, by means of a headphone connection on the prototype circuit, it reaches a heart sound level, that is, an electrical signal, which can be heard by a headset used by the user. They carried the heart rate of the fetus from the doppler device to the android platform in their study called "Mobile - FHR Assessment Using Android Platform" [2]. Chalmers University of Technology

${ }^{1}$ Department of Software Engineering, Faculty of Engineering, Aksaray University, Aksaray- 68100, Türkiye, ORCID ID: 0000-0002-1622-9059 * Corresponding Author Email: emreavuclu@aksaray.edu.tr

student Susanne Andersson's "Acceleration and Deceleration Detection and Baseline Estimation" in her master's thesis described the interpretation of electronic signals from any Doppler instrument using the Dawes-Redman algorithm [3]. Finland-based software company Odosoft born "UnbornHeart" developed the mobile application. It has the feature of listening to the heart rate of the fetus and the heartbeat sound of the fetus to the expectant mother [4]. The most important criterion in the evaluation of fetal health is the measurement and interpretation of FHR and UC values. These measurements can be made in clinical or hospital environment with advanced technology and high cost Non-Stress Test (NST) devices. In order to apply NST measurement, the pregnant individual must make an appointment with the health institutions in advance, reach the clinic or hospital environment and connect to the NST device for at least 20 minutes. Depending on the status of the pregnancy and the risk group, these procedures should be repeated periodically until the end of pregnancy.

Several studies have been carried out using ML algorithms related to FHR. Cardiotocogram classification [5], fetal monitoring [6], using nonlinear features for FHR classification [7], Fuzzy analysis of birth outcome characteristics to better automate fetal status assessment, classification of cardiotocogram data to predict fetal risks [8-9]. CTG has taken a pivotal role in the development of objective measures as a function of CTG signals [10], particularly within the machine learning community [11-21], studies representing non-visual patterns found in FHR [22-25]. Blinx et al. compared DT, Neural Network and Discriminant Analysis. The ANN classifier achieves an overall accuracy of $97.78 \%$ [26].

In this important issue, this study was carried out so that the classification and diagnosis process can be performed automatically. The aim of this study is to develop a hybrid model for faster and more accurate interpretation of FHR results. In this 
way, errors and delays caused by the negativity will be eliminated.

\section{Material and Methods}

MLAs can be defined as brains that allow machines to learn, making them smarter. Exposing these algorithms to new data and experiences on a regular basis; It allows great things to be accomplished in various tasks related to classification, predictive modeling and analysis of data.

The learning process begins with inputting training data such as examples, direct experience, instructions, observations into the chosen algorithm to look for patterns in the data. To test whether this algorithm works correctly, new input data is added to the machine learning algorithm. The prediction and results are then checked.

\subsection{Algorithms}

$\mathrm{k}-\mathrm{NN}$ is an algorithm that uses training datasets to find the $\mathrm{k}$ closest data points in some dataset. $\mathrm{k}-\mathrm{NN}$ is a supervised MLA used for both regression and classification problems. This algorithm first stores and defines the distance between all inputs in the data, choosing the closest input to the query and outputs.

The DT algorithm is a type of supervised machine learning. It is used to solve regression and classification problems. The goal is to leverage a decision tree to move from observations to processing results.

NB algorithm, which calculates the probability of an item falling into a certain category using the conditional probability rule, is known as supervised MLA.

In the SVM algorithm, data is classified according to the degree of polarity that can go beyond the $\mathrm{X} / \mathrm{Y}$ estimation. SVM is categorized among supervised MLAs and is primarily used for classification and regression analysis. The algorithm works by creating models that assign new instances and data to a category.

\subsection{Dataset and Features}

The dataset contains information including measurements of FHR and UC features in cardiotocograms classified by obstetricians [27]. Records of 2126 fetal cardiotocograms (CTGs) were automatically processed and the measurement of diagnostic features required was recorded. Classification in the database was performed according to both morphological pattern (A, B, C....) and fetal status $(\mathrm{N}, \mathrm{S}, \mathrm{P})$. As a result, the dataset can be used in any study that will be used for 10-class or 3-class experiments. Dataset attribute information shows following in Figure 1 [28].

\begin{tabular}{ll}
\hline Feature & Descriptions \\
\hline LB & FHR baseline (beats per minute) \\
AC & \# of accelerations per second \\
FM & \# of fetal movements per second \\
UC & \# of uterine contractions per second \\
DL & \# of light decelerations per second \\
DS & \# of severe decelerations per second \\
DP & \# of prolongued decelerations per second \\
ASTV & percentage of time with abnormal short term variability \\
MSTV & mean value of short term variability \\
ALTV & percentage of time with abnormal long term variability \\
MLTV & mean value of long term variability \\
Width & width of FHR histogram \\
Min & minimum of FHR histogram \\
Max & maximum of FHR histogram \\
Nmax & \# of histogram peaks \\
Nzeros & \# of histogram zeros \\
Mode & histogram mode \\
Mean & histogram mean \\
Median & histogram median \\
Variance & histogram variance \\
Tendency & histogram tendency \\
NSP & fetal state class code (N=normal; S=suspect; P=pathologic) \\
\hline
\end{tabular}

Figure 1. Dataset information

\section{Experimental Results}

The structure of the developed hybrid model is as shown in Figure 2. Different hybrid models were tried during the studies, but this model provided the exact diagnosis rate. In the proposed hybrid model, operations are carried out with the machine learning algorithm, which always achieves the highest accuracy, and the next step is taken.

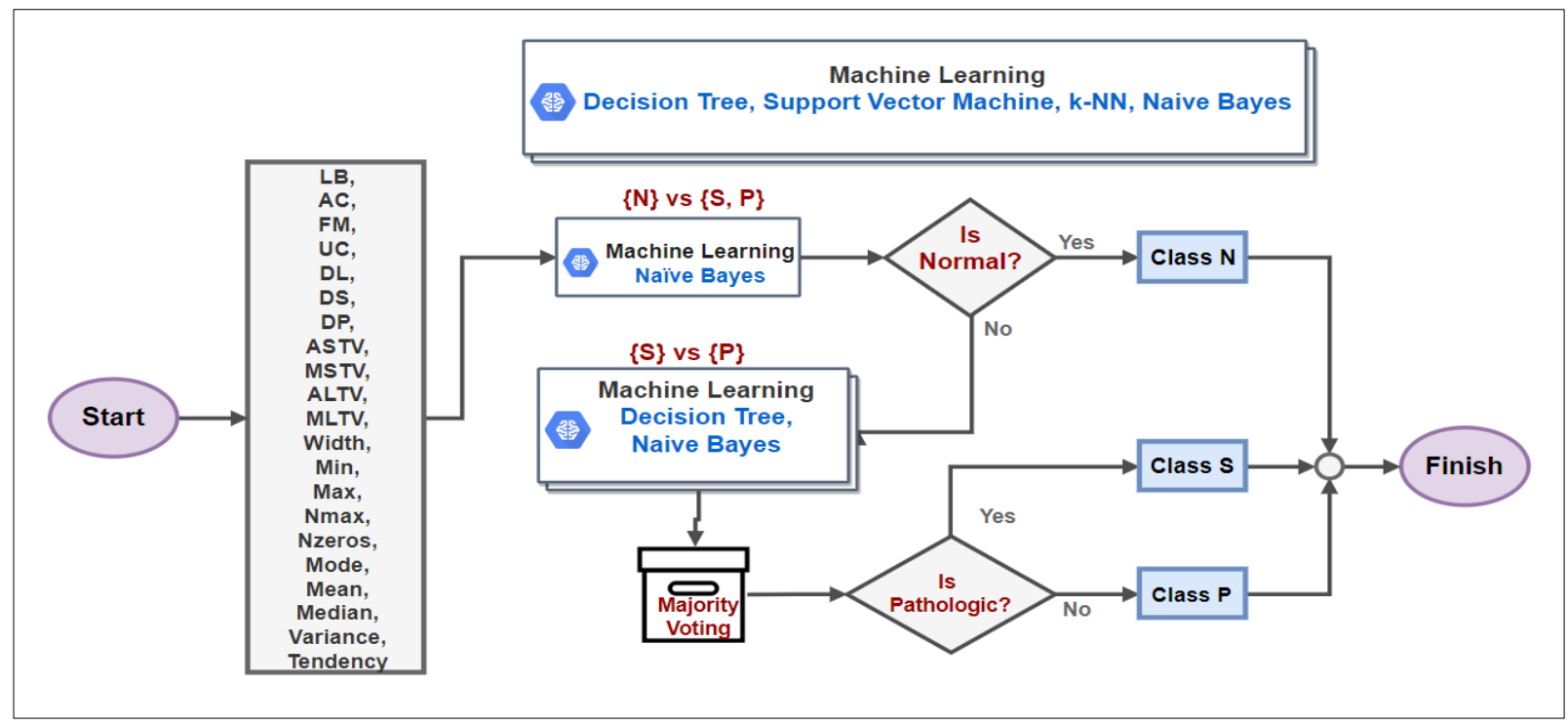

Figure 2. Proposed hybrid model 
The confusion matrix shows the current state of the data and the number of correct and incorrect predictions of our classification model. The statistical measurements used in this study are shown in the Figure 3.

$65 \%$ of the data in the database was used for training and $35 \%$ for the test. The classification and test results, which were initially performed without any hybrid model, are as follows in Table 1, Table 2 and Table 3 . The statistical results obtained from the k-NN ML algorithm without the hybrid model are shown in Table 2 (1, 2, 3, 4, 5, 6, 7, 8, 9 = TPR, SPC, PPV, NPV, FPR, FNR, ACC, MCC, FM for all tables).

\begin{tabular}{|c|c|c|c|}
\hline $\begin{array}{l}\text { Sensitivity or } \\
\text { True Positive Rate }\end{array}$ & $T P R=\frac{T P}{T P+F N}$ & $\begin{array}{l}\text { Dice Similarity } \\
\text { Coefficient }\end{array}$ & $D S C=\frac{2 T P}{2 T P+F P+F N}$ \\
\hline $\begin{array}{l}\text { Specificity or } \\
\text { True Negative Rate }\end{array}$ & $T N R=\frac{T N}{T N+F P}$ & \multirow[b]{2}{*}{ Accuracy } & \multirow[b]{2}{*}{$A C C=\frac{T P+T N}{T P+T N+F P+F N}$} \\
\hline $\begin{array}{l}\text { Precision or } \\
\text { Positive Predictive } \\
\text { Value }\end{array}$ & $P P V=\frac{T P}{T P+F P}$ & & \\
\hline $\begin{array}{l}\text { Negative Predictive } \\
\text { Value }\end{array}$ & $N P V=\frac{T N}{T N+F N}$ & F-Measurements & $F M=\frac{2}{\frac{1}{T P R}+\frac{1}{P P V}}$ \\
\hline False Positive Ratio & $F P R=\frac{F P}{T N+F P}$ & \multirow{2}{*}{\multicolumn{2}{|c|}{$\begin{array}{l}\text { Matthews Correlation Coefficient } \\
M C C=\frac{T P \times T N-F P \times F N}{\sqrt{(T P+F P)(T P+F N)(T N+F P)(T N+F N)}}\end{array}$}} \\
\hline False Negative Ratio & $F N R=\frac{F N}{I P+F N}$ & & \\
\hline
\end{tabular}

Figure 3. Statistical measurements.

Table 1. k-NN ML algorithm statistical results

\begin{tabular}{|c|c|c|c|c|c|c|c|c|}
\hline & 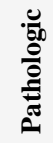 & $\begin{array}{l}\overline{\mathfrak{Z}} \\
\text { है } \\
\text { Z }\end{array}$ & 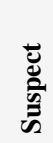 & & $\hat{E}$ & $\hat{E}$ & 㒰 & Z \\
\hline Pathologic & 23 & 64 & 3 & Pathologic & 23 & 67 & 7 & 660 \\
\hline Normal & 6 & 531 & 89 & Normal & 531 & 95 & 87 & 44 \\
\hline Suspect & 1 & 23 & 17 & Suspect & 17 & 24 & 92 & 624 \\
\hline
\end{tabular}

\begin{tabular}{|c|c|c|c|c|c|c|c|c|c|}
\cline { 2 - 9 } \multicolumn{1}{c|}{} & $\mathbf{1}$ & $\mathbf{2}$ & $\mathbf{3}$ & $\mathbf{4}$ & $\mathbf{5}$ & $\mathbf{6}$ & $\mathbf{7}$ & $\mathbf{8}$ & $\mathbf{9}$ \\
\hline Pathologic & 0.77 & 0.91 & 0.26 & 0.99 & 0.09 & 0.01 & 0.9 & 0.41 & 0.38 \\
\hline Normal & 0.86 & 0.32 & 0.85 & 0.34 & 0.68 & 0.66 & 0.76 & 0.18 & 0.85 \\
\hline Suspect & 0.16 & 0.96 & 0.41 & 0.87 & 0.04 & 0.13 & 0.85 & 0.18 & 0.23 \\
\hline
\end{tabular}

It is also shown graphically in Figure 4.

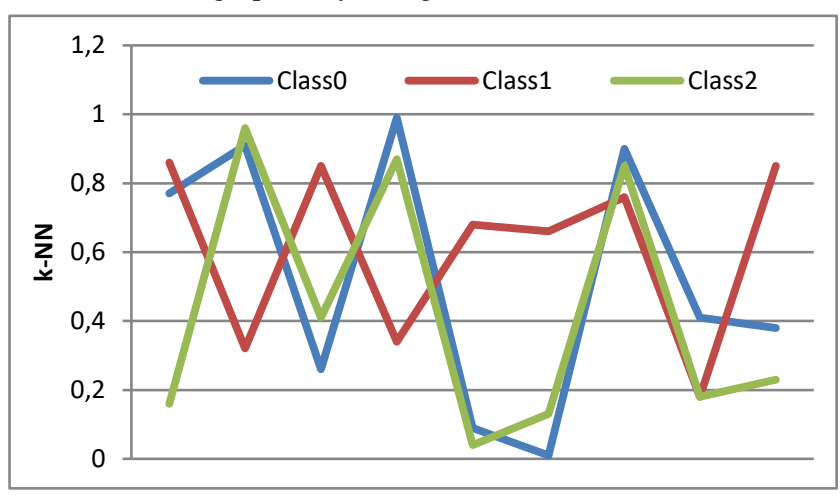

Figure 4. k-NN ML algorithm statistical results

The statistical results obtained from the DT ML algorithm without the hybrid model are shown in Table 2 .
Table 2. Statistical results of DT ML algorithm

\begin{tabular}{|c|c|c|c|c|c|c|c|c|}
\hline & 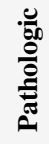 & 己⿱艹 & 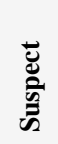 & & 官 & 宝 & Z & Z \\
\hline \multirow{3}{*}{$\begin{array}{c}\text { Pathologic } \\
\text { Normal } \\
\text { Suspect }\end{array}$} & 90 & 0 & 0 & \multirow{3}{*}{$\begin{array}{c}\text { Pathologic } \\
\text { Normal } \\
\text { Suspect }\end{array}$} & 90 & 0 & 0 & 667 \\
\hline & 0 & 480 & 146 & & 480 & 146 & 10 & 121 \\
\hline & 0 & 10 & 31 & & 31 & 10 & 146 & 570 \\
\hline
\end{tabular}

\begin{tabular}{|c|c|c|c|c|c|c|c|c|c|}
\cline { 2 - 9 } \multicolumn{1}{c|}{} & $\mathbf{1}$ & $\mathbf{2}$ & $\mathbf{3}$ & $\mathbf{4}$ & $\mathbf{5}$ & $\mathbf{6}$ & $\mathbf{7}$ & $\mathbf{8}$ & $\mathbf{9}$ \\
\hline Pathologic & 1 & 1 & 1 & 1 & 0 & 0 & 1 & 1 & 1 \\
\hline Normal & 0.98 & 0.45 & 0.77 & 0.92 & 0.55 & 0.08 & 0.79 & 0.55 & 0.86 \\
\hline Suspect & 0.18 & 0.98 & 0.76 & 0.8 & 0.02 & 0.2 & 0.79 & 0.3 & 0.28 \\
\hline
\end{tabular}

It is also shown graphically in Figure 5.

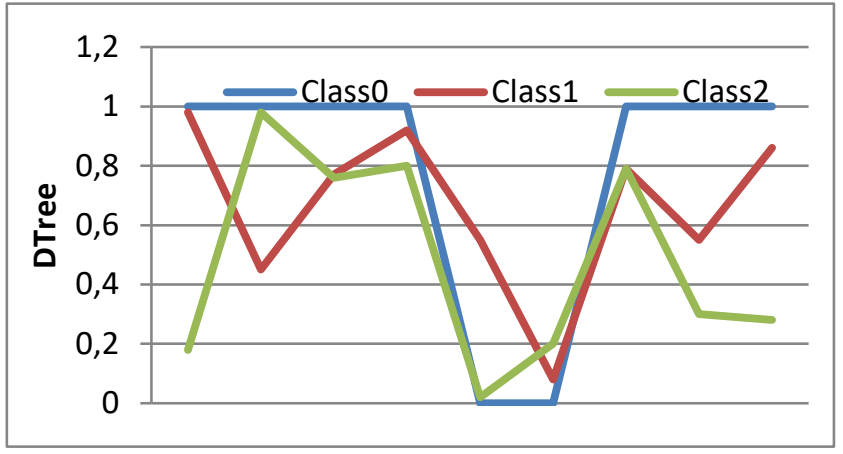

Figure 5. DT ML algorithm statistical results

The statistical results obtained from the NB ML algorithm without the hybrid model are shown in Table 3.

Table 3. NB ML algorithm statistical results

\begin{tabular}{|c|c|c|c|c|c|c|c|c|c|c|}
\hline & 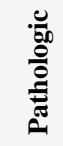 & 范 & 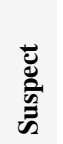 & & & & $\hat{\theta}$ & t) & $Z_{X}$ & Z \\
\hline Pathologic & 90 & 0 & 0 & & \multirow{3}{*}{\multicolumn{2}{|c|}{$\begin{array}{c}\text { Pathologic } \\
\text { Normal } \\
\text { Suspect }\end{array}$}} & 90 & 0 & 0 & 667 \\
\hline Normal & 0 & 613 & 13 & & & & 613 & 13 & 3 & 128 \\
\hline \multirow[t]{2}{*}{ Suspect } & 0 & 3 & 38 & & & & 38 & 3 & 13 & 703 \\
\hline & 1 & 2 & 3 & 4 & 5 & 6 & 7 & & 8 & 9 \\
\hline Pathologic & 1 & 1 & 1 & 1 & 0 & 0 & 1 & & 1 & 1 \\
\hline Normal & 1 & 0.91 & 0.98 & 0.98 & 0.09 & 0.02 & 0.98 & & .93 & 0.99 \\
\hline Suspect & 0.75 & 1 & 0.93 & 0.98 & 0 & 0.02 & 0.98 & & .82 & 0.83 \\
\hline
\end{tabular}

It is also shown graphically in Figure 6.

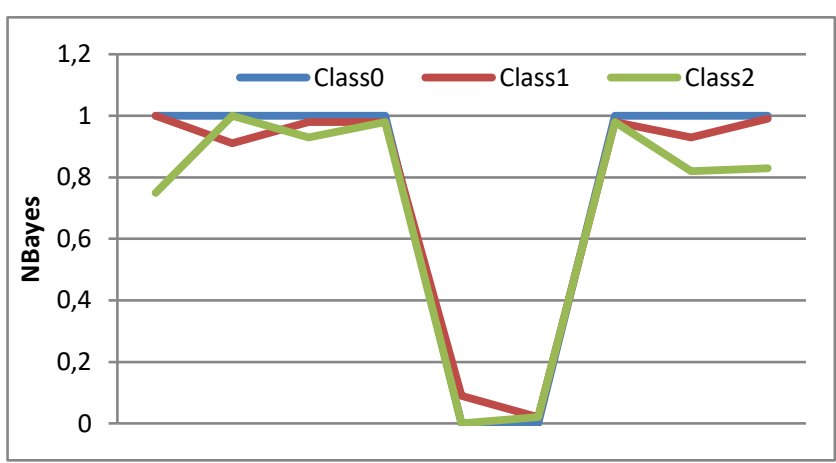

Figure 6. NB ML algorithm statistical results 
The statistical results obtained from the SVM ML algorithm without the hybrid model are shown in Table 4.

Table 4. Statistical results of SVM ML algorithm

\begin{tabular}{|c|c|c|c|c|c|c|c|c|c|}
\hline & 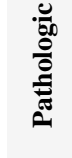 & $\begin{array}{l}\overline{\text { J }} \\
\bar{\Xi} \\
\bar{Z}\end{array}$ & 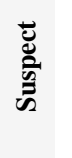 & & & $\hat{F}$ & $\overrightarrow{\mid}$ & 至 & Z \\
\hline Pathologic & 15 & 75 & 0 & \multirow{3}{*}{\multicolumn{2}{|c|}{$\begin{array}{c}\text { Pathologic } \\
\text { Normal } \\
\text { Suspect }\end{array}$}} & 15 & 75 & 0 & 667 \\
\hline Normal & 0 & 578 & 48 & & & 578 & 48 & 101 & 30 \\
\hline \multirow[t]{2}{*}{ Suspect } & 0 & 26 & 15 & & & 15 & 26 & 48 & 668 \\
\hline & 1 & 2 & 3 & 4 & 5 & 6 & 7 & 8 & 9 \\
\hline Pathologic & 1 & 0.9 & 0.17 & 1 & 0.1 & 0 & 0.9 & 0.39 & 0.29 \\
\hline Normal & 0.85 & 0.38 & 0.92 & 0.23 & 0.62 & 0.77 & 0.8 & 0.19 & 0.89 \\
\hline Suspect & 0.24 & 0.96 & 0.37 & 0.93 & 0.04 & 0.07 & 0.9 & 0.24 & 0.29 \\
\hline
\end{tabular}

It is also shown graphically in Figure 7.

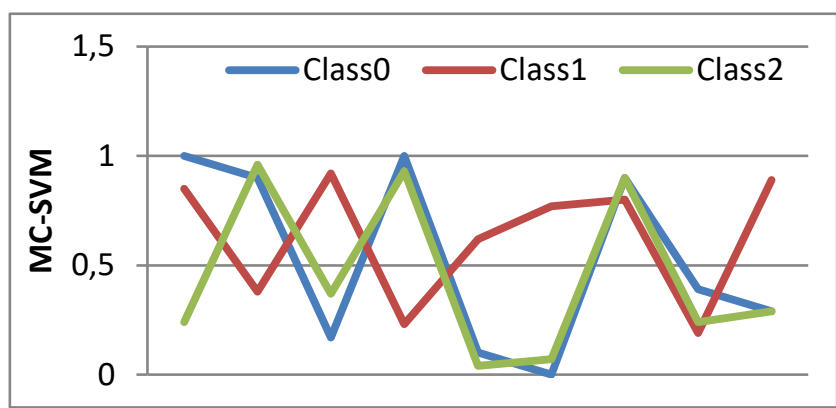

Figure 7. SVM ML algorithm statistical results

Table 5. k-NN algorithm statistical results

\begin{tabular}{|c|c|c|c|c|c|c|c|c|c|}
\hline & 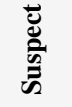 & 蹗 & & & & $\hat{=}$ & 宝 & $z$ & $Z$ \\
\hline Suspect & 23 & 70 & & & Suspe & et 23 & 70 & 2 & 39 \\
\hline \multirow[t]{2}{*}{ Normal } & 2 & 39 & & & Norm & al 39 & 2 & 70 & 23 \\
\hline & 1 & 2 & 3 & 4 & 5 & 6 & 7 & 8 & 9 \\
\hline Suspect & 0.92 & 0.36 & 0.25 & 0.95 & 0.64 & 0.05 & 0.46 & 0.23 & 0.39 \\
\hline Normal & 0.36 & 0.92 & 0.95 & 0.25 & 0.08 & 0.75 & 0.46 & 0.23 & 0.52 \\
\hline
\end{tabular}

The training and test scores obtained from the hybrid model ML algorithms are given below Table 5, Table 6 , Table 7, respectively. The statistical results obtained from the k-NN after the generated hybrid model are shown in Table 5.

It is also shown graphically in Figure 8.

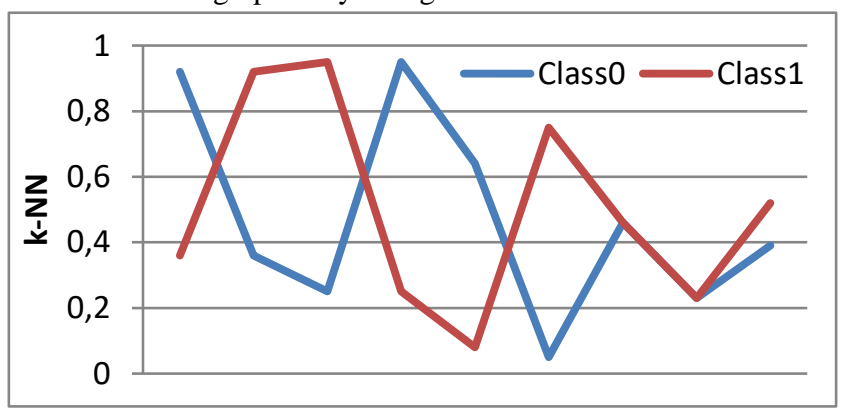

Figure 8. k-NN ML algorithm statistical results
The statistical results obtained from the DT after the generated hybrid model are shown in Table 6.

Table 6. DT algorithm statistical results

\begin{tabular}{|c|c|c|c|c|c|c|c|c|c|}
\hline & \multicolumn{2}{|c|}{ 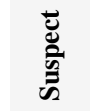 } & 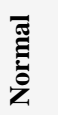 & & & 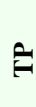 & 宝 & $Z_{\mathbf{I}}$ & Z \\
\hline Suspect & & & ) & \multicolumn{2}{|c|}{ Suspect } & 93 & 0 & 0 & 41 \\
\hline \multicolumn{2}{|l|}{ Normal } & 0 & 41 & \multicolumn{2}{|c|}{ Normal } & 41 & 0 & 0 & 93 \\
\hline & 1 & 2 & 3 & 4 & 5 & 6 & 7 & 8 & 9 \\
\hline Suspect & 1 & 1 & 1 & 1 & 0 & 0 & 1 & 1 & 1 \\
\hline Normal & 1 & 1 & 1 & 1 & 0 & 0 & 1 & 1 & 1 \\
\hline
\end{tabular}

It is also shown graphically in Figure 9.

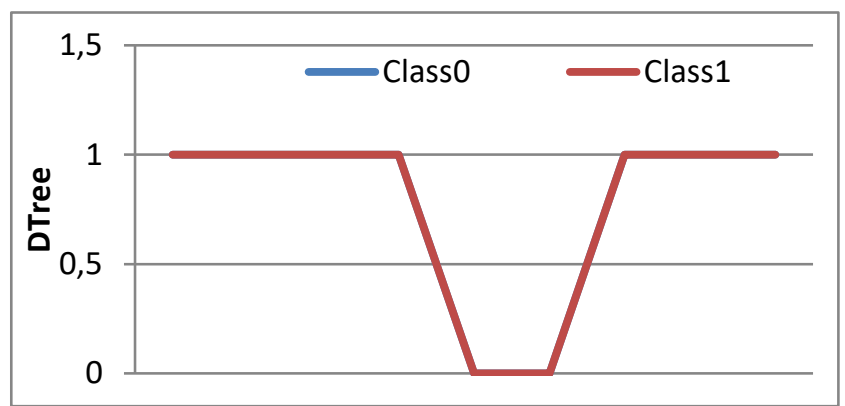

Figure 9. DT ML algorithm statistical results

The statistical results obtained after the generated hybrid model NB are shown in Table 7.

Table 7. NB algorithm statistical results

\begin{tabular}{|c|c|c|c|c|c|c|c|}
\hline & 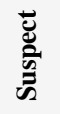 & $\begin{array}{l}\bar{\pi} \\
\overline{\mathbf{z}} \\
\overline{\mathbf{z}}\end{array}$ & & $\hat{Z}$ & $\overrightarrow{\mid}$ & Z & Z \\
\hline Suspect & 93 & 0 & Suspect & 93 & 0 & 0 & 41 \\
\hline Normal & 0 & 41 & Normal & 41 & 0 & 0 & 93 \\
\hline
\end{tabular}

\begin{tabular}{|l|l|l|l|l|l|l|l|l|l|}
\cline { 2 - 10 } \multicolumn{1}{c|}{} & $\mathbf{1}$ & $\mathbf{2}$ & $\mathbf{3}$ & $\mathbf{4}$ & $\mathbf{5}$ & $\mathbf{6}$ & $\mathbf{7}$ & $\mathbf{8}$ & $\mathbf{9}$ \\
\hline Suspect & 1 & 1 & 1 & 1 & 0 & 0 & 1 & 1 & 1 \\
\hline Normal & 1 & 1 & 1 & 1 & 0 & 0 & 1 & 1 & 1 \\
\hline
\end{tabular}

It is also shown graphically in Figure 10.

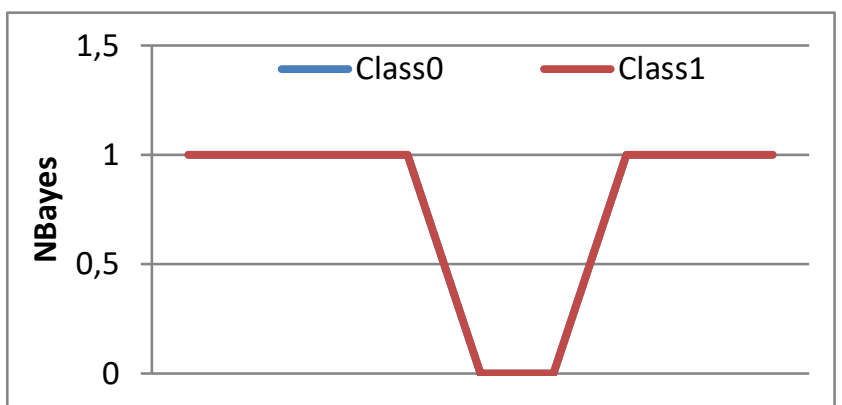

Figure 10. NB ML algorithm statistical results 
The statistical results obtained from the SVM after the hybrid model generated are as shown in Table 8 .

Table 8. SVM algorithm statistical results

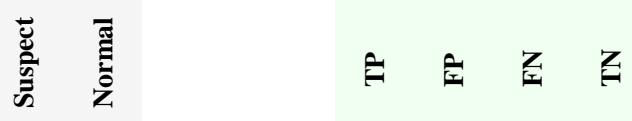

\begin{tabular}{cccccccc} 
Suspect & 6 & 87 & Suspect & 6 & 87 & 0 & 41 \\
Normal & 0 & 41 & Normal & 41 & 0 & 87 & 6 \\
\hline
\end{tabular}

\begin{tabular}{|c|c|c|c|c|c|c|c|c|c|}
\cline { 2 - 10 } \multicolumn{1}{c|}{} & $\mathbf{1}$ & $\mathbf{2}$ & $\mathbf{3}$ & $\mathbf{4}$ & $\mathbf{5}$ & $\mathbf{6}$ & $\mathbf{7}$ & $\mathbf{8}$ & $\mathbf{9}$ \\
\hline Suspect & 1 & 0.32 & 0.06 & 1 & 0.68 & 0 & 0.35 & 0.14 & 0.12 \\
\hline Normal & 0.32 & 1 & 1 & 0.06 & 0 & 0.94 & 0.35 & 0.14 & 0.49 \\
\hline
\end{tabular}

It is also shown graphically in Figure 11.

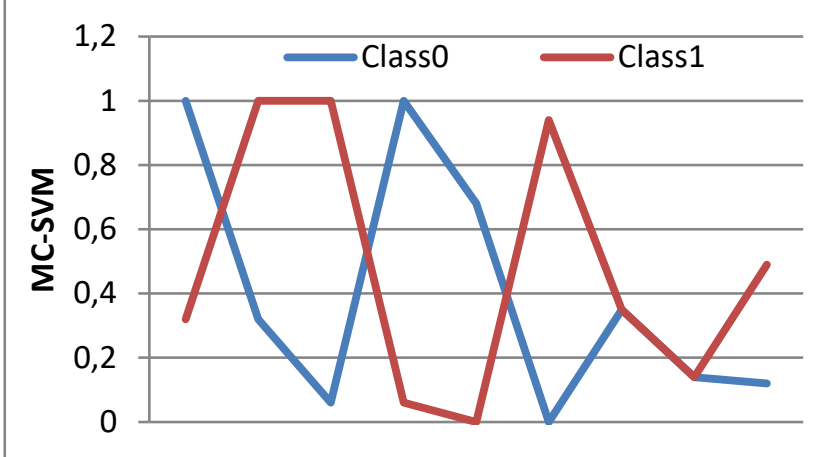

Figure 11. SVM ML algorithm statistical results

ROC curves obtained from MLA as a result of the application of normal and hybrid models are given in Table 9.

Table 9. ROC curve results

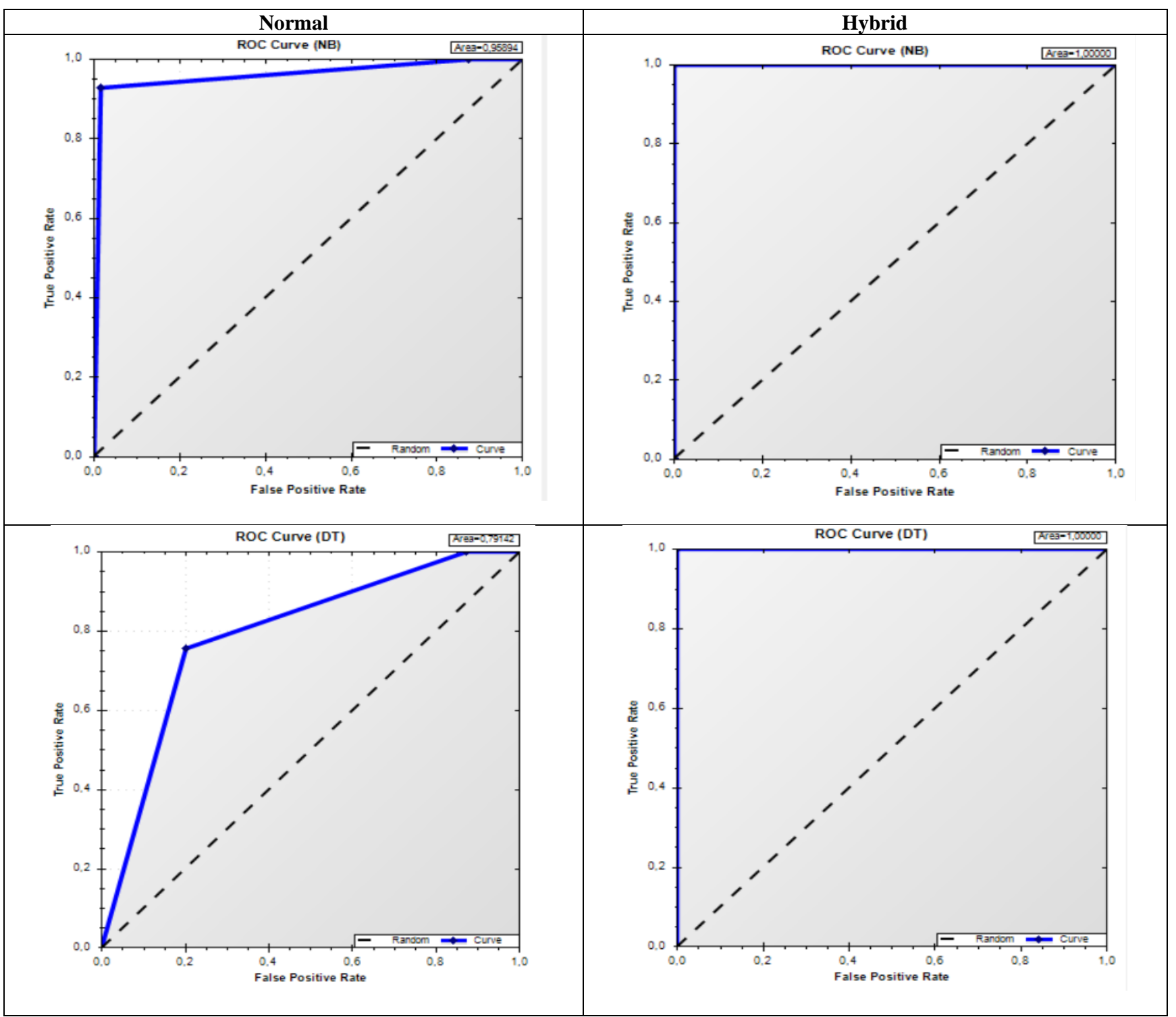

When looking at the Table 9, it is seen that the ROC curve values of the proposed hybrid result increase. Accuracy values obtained as normal and hyrid results are shown in Table 10. 
Table 10. Accuracy values obtained from normal and hybrid models

\begin{tabular}{ccccc}
\hline & \multicolumn{2}{c}{ Normal } & \multicolumn{2}{c}{ Hybrid } \\
\hline & Train score & Test score & Train score & $\begin{array}{c}\text { Test } \\
\text { score }\end{array}$ \\
\cline { 2 - 5 } k-NN & 1 & 0.74636 & 1 & 0.47014 \\
DT & 1 & 0.79395 & 1 & 1 \\
NB & 0.97808 & 0.97886 & 0.994065 & 1 \\
SVM & 0.92037 & 0.771466 & 0.9020771 & 0.440298 \\
\hline
\end{tabular}

Accuracy values increased as a result of the proposed Hybrid model. The training and test data obtained after the normal classification (without hybrid model), the first stage (with hybrid model) and the final stage (with hybrid model) are as shown in Table 11

Table 11. Step-by-step classification results (hybrid model)

\begin{tabular}{llll}
\hline \multicolumn{4}{c}{ Normal classification result } \\
\hline k-NN & DT & NB & SVM \\
\hline Train Score $\% 100$ & $\% 100$ & $\% 97.81$ & $\% 84.08$ \\
Test Score $\% 75.43$ & $\% 79.39$ & $\% 97.89$ & $\% 80.32$ \\
\hline \multicolumn{4}{c}{ Final classification result } \\
\hline k-NN & DT & NB & SVM \\
\hline Train Score $\% 100$ & $\% 100$ & $\% 99.41$ & $\% 77,15$ \\
Test Score $\% 46.27$ & $\% 100$ & $\% 100$ & $\% 35,07$ \\
\hline
\end{tabular}

Looking at the Table s above, it will be seen that the hybrid model has achieved its goal by increasing the classification and test results at each step. The proposed hybrid model reached better results than other studies in the literature. In Table 12, comparisons of other methods and the proposed hybrid model are given.

Table 12. Comparison with other method

\begin{tabular}{|c|c|c|c|c|c|c|c|}
\hline \multicolumn{8}{|c|}{ Other Methods (\%) } \\
\hline & 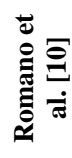 & 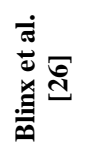 & 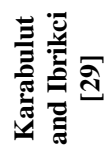 & 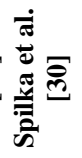 & 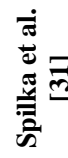 & $\frac{\tilde{\sigma}}{\tilde{\Xi}}$ & 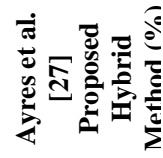 \\
\hline Accuracy & 77 & - & 93.97 & 95.01 & - & - & 100 \\
\hline Sensitivity & 93 & - & 91.58 & - & 72 & 73.4 & 100 \\
\hline Specificity & 82 & - & 95.79 & - & 78 & 76.3 & 100 \\
\hline $\begin{array}{l}\text { Overall } \\
\text { classifier }\end{array}$ & - & 97.78 & - & - & - & - & 100 \\
\hline Train Score & - & - & - & - & - & - & 100 \\
\hline Test Score & - & - & - & - & - & - & 100 \\
\hline
\end{tabular}

\section{4. . Conclusions}

CTG recordings are widely used in pregnancy today because CTG provides important information about the physiological health of the fetus. In this study, a hybrid model was proposed, which allows automatic, rapid and complete diagnosis of CTG recordings. With this model, any discomfort in the fetus can be detected instantly with the diagnostic results to be given automatically. In this way, the necessary precautions can be taken by determining the negativities in pregnancy. While the average classification rates were $95.4725 \%$ training and $83.2575 \%$ test results on average, with this proposed hybrid model, this average value was obtained as $98.3925 \%$ training and $94.4175 \%$ test results. With the hybrid model created in the study, a $34 \%$ improvement was achieved for accurate diagnosis. Sensitivity and Specificity ratios were obtained as $100 \%$ with the proposed hybrid model. In general, the classification results have increased and reached a level that can be fully diagnosed.

\section{References}

[1] Tekin gündüz, S., kurtuldu, A., \& Türkkan, I. Ş. I. K. (2017). Sağlık Hizmetlerinde Eşitsizlik ve Etik. Aksaray Üniversitesi İktisadi ve İdari Bilimler Fakültesi Dergisi, 8(4), 32-43.

[2] Zach, L., Chudáček, V., Kužílek, J., Spilka, J., Huptych, M., Burša, M., \& Lhotská, L. (2011). Mobile CTG_-Fetal heart rate assessment using Android platform. In Computing in Cardiology (pp. 249-252). IEEE.

[3] Andersson, S. U. S. A. N. N. E. (2011). Acceleration and deceleration detection and baseline estimation. Göteborg: Chalmers University of Technology.

[4] UnbornHeart, http://www.unbornheart.com/, Accessed Date:14.5.2018.

[5] A. Pinas and E. Chandraharan, (2016). Continuous cardiotocography during labour: Analysis, classification and management, Best Practice \& Research Clinical Obstetrics \& Gynaecology, vol. 30, pp. 33-47.

[6] D. Ayres-de-Campos, C. Y. Spong, E. (2015). Chandraharan, and F. I. F. M. E. C. Panel, FIGO consensus guidelines on intrapartum fetal monitoring: Cardiotocography, Int J Gynaecol Obstet, vol. 131, pp. 13-24.

[7] J. Spilka, V. Chudáček, M. Koucký, L. Lhotská, M. Huptych, P. Janků, et al., (2012). Using nonlinear features for fetal heart rate classification, Biomedical Signal Processing and Control, vol. 7, pp. 350-357.

[8] R. Czabanski, M. Jezewski, K. Horoba, J. Jezewski, and J. Leski, (2016). Fuzzy Analysis of Delivery Outcome Attributes for Improving the Automated Fetal State Assessment, Applied Artificial Intelligence, vol. 30, pp. 556-571.

[9] H. Sahin and A. Subasi, (2015). Classification of the cardiotocogram data for anticipation of fetal risks using machine learning techniques, Applied Soft Computing, vol. 33, pp. 231-238.

[10] M. Romano, P. Bifulco, M. Ruffo, G. Improta, F. Clemente, M. Cesarelli, (2016). Software for computerised analysis of cardiotocographic traces, Comput. Methods Programs Biomed., 124, pp. 121-137.

[11] J. Kessler, D. Moster, S. Albrechfsen, (2014). Delay in intervention increases neonatal morbidity in births monitored with Cardiotocography and ST-waveform analysis, Acta Obs. Gynecol. Scand., 93 (2), pp. 175-181.

[12] P.A. Warrick, E.F. Hamilton, D. Precup, R.E. Kearney, (2010). Classification of normal and hypoxic fetuses from systems modeling of intrapartum cardiotocography, IEEE Trans. Biomed. Eng., 57 (4), pp. 771-779.

[13] A. Pinas, E. Chadraharan, (2016). Continuous Cardiotocography during labour: analysis, classification and management, Best. Pract. Res. Clin. Obstet. Gynaecol., 30, pp. 33-47.

[14] P.A. Warrick, E.F. Hamilton, D. Precup, R.E. Kearney (2010). Classification of normal and hypoxic fetuses from systems modeling of intrapartum cardiotocography, IEEE Trans. Biomed. Eng., 57 (4), pp. 771-779.

[15] H. Sahin, A. Subasi, (2015). Classification of the cardiotocogram data for anticipation of fetal risks using machine learning techniques, Appl. Soft Comput., 33, pp. 231-238

[16] H. Ocak, H.M. Ertunc, (2013). Prediction of fetal state from the cardiotocogram recordings using adaptive neuro-fuzzy inference systems, Neural comput. Appliations, 22 (6), pp. 1583-1589.

[17] C. Rotariu, A. Pasarica, G. Andruseac, H. Costin, D. (2014). Nemescu Automatic analysis of the fetal heart rate variability and uterine contractions, IEEE Electrical and Power Engineering, pp. 
553-556.

[18] C. Rotariu, A. Pasarica, H. Costin, D,. Nemescu (2014). Spectral analysis of fetal heart rate variability associated with fetal acidosis and base deficit values, International Conference on Development and Application Systems, pp. 210-213.

[19] K. Maeda, (2014). Modalities of fetal evaluation to detect fetal compromise prior to the development of significant neurological damage, J. Obstet. adn Gynaecol. Res., 40 (10), pp. 2089-2094.

[20] H. Ocak, (2013). A medical decision support system based on support vector machines and the genetic algorithm for the evaluation of fetal well-being. J. Med. Syst., 37 (2), p. 9913.

[21] T. Peterek, P. Gajdos, P. Dohnalek, J. Krohova (2014). Human fetus Health classification on cardiotocographic data using random forests, Intelligent Data Analysis and its Applications, pp. 189-198.

[22] C. Buhimschi, M.B. Boyle, G.R. Saade, R.E. (1998). Garfield Uterine activity during pregnancy and labor assessed by simultaneous recordings from the myometrium and abdominal surface in the rat, Am. J. Obstet. Gynecol., 178 (4), pp. 811-822.

[23] C. Buhimschi, M.B. Boyle, R.E. Garfield, (1997). Electrical activity of the human uterus during pregnancy as recorded from the abdominal surface, Obstet. Gynecol., 90 (1), pp. 102-111.

[24] C. Buhimschi, R.E. (1996). Garfield Uterine contractility as assessed by abdominal surface recording of electromyographic activity in rats during pregnancy, Am. J. Obstet. Gynecol., 174 (2), pp. 744-753.

[25] J.S. Richman, J.R. Moorman (2000). Physiological time-series analysis using approximate entropy and sample entropy, Am. J. Physiol. - Hear. Circ. Physiol., 278 (6).

[26] E. Blinx, K.G. Brurberg, E. Reierth, L.M. Reinar, P. Oian ST (2016). Waveform analysis versus Cardiotocography alone for intrapartum fetal monitoring: a systematic review and meta-analysis of randomized trials, Acta Obstet. Gynancelogica Scand., 95 (1), pp. 16-27

[27] D Ayres-de Campos, J Bernardes, A Garrido, J Marques-de-Sá, L Pereira-Leite, (2000). SisPorto 2.0 A program for Automated Analysis of Cardiotocograms. J Matern Fetal Med. 5:311-318.

[28] Web site: https://archive.ics.uci.edu/ml/datasets/Cardiotocography\#, Access date:10.7.2019.

[29] E.M. Karabulut, T. Ibrikci, (2014). Analysis of cardiotocogram data for fetal distress determination by decision tree based adaptive boosting approach, J. Comput. Commun., 2 (9), pp. 32-37.

[30] J. Spilka, G. Georgoulas, P. Karvelis, V. Chudacek (2014). Discriminating normal from 'Abnormal' pregnancy cases using an automated FHR evaluation, Method Artif. Intell. Methods Appl., 8445, pp. 521-531.

[31] J. Spilka, V. Chudacek, M. Koucky, L. Lhotska, M. Huptych, P. Janku, G. Georgoulas, C. Stylios, (2012). Using nonlinear features for fetal heart rate Classification, Biomed. Signal Process. Control, 7 (4), pp. 350-357.

[32] Paul Fergus, M. Selvaraj, Carl Chalmers, (2018). Machine learning ensemble modelling to classify caesarean section and vaginal delivery types using Cardiotocography traces, Computers In Biology And Medicine, 93, pp. 7-16. 